\title{
Internet of Things (IoT): The Most Up-To-Date Challenges, Architectures, Emerging Trends and Potential Opportunities
}

\author{
Emrah Irmak \\ Research Fellow \\ Biomedical Engineering Department \\ Karabuk University, Karabuk, Turkey
}

\author{
Mehmet Bozdal \\ $\mathrm{PhD}$ Researcher \\ IVHM Centre \\ Cranfield University, Cranfield, UK
}

\begin{abstract}
Internet of Things (IoT) is nowadays the most profound buzzword in Information Technology science. IoT is the evolution of Information Technology which aims to build a mutual infrastructure that integrates, connects and telecommunicates every 'Things' (Objects) with each other on the face of the earth. This interconnected infrastructure provides humans with fully control of things. Projections and potential estimates about incomings of IoT are spectacular for future directions. IoT encompasses such a wide range of spectrum that its influences are anyone's guess. Not only the profits of IoT but also the forfeits and fears of IoT are unpredictable for yet. However, with the increase in research, academic studies and technological developments the atmosphere will be clear for IoT. This research paper proposes a novel comprehensive reference source for those who are interested in IoT, ubiquitous sensing, pervasive computing and smart objects. The paper explains IoT emergence and IoT history in detail, current IoT usage areas, the most up-to-date potential opportunities and future IoT directions, overall IoT architecture and well-liked architectures, security and privacy concerns about IoT. The latest ongoing IoT projects are discussed and the latest burning issues are presented. Critical and turning points of IoT are given in tabular. As compared to similar survey papers in the area, to the best of our knowledge contributions of this paper are unique.
\end{abstract}

\section{General Terms}

Internet of Things, Ubiquitous Computing, Pervasive Computing, Smart Environments, Security and Privacy

\section{Keywords}

IoT, Ubiquitous Computing, IoT Security

\section{INTRODUCTION}

\subsection{Definition, Concept And Importance}

There are two very big revolutions for human beings for the recent past. The first one is The Internet which leads forth interconnection of people at an unpredictable scale and level. The second one is on its way, The Internet of Things. "The Internet of Things has the potential to change the world, just as the Internet did. Maybe even more so." says Kevin Ashton who is an innovator and consumer sensor expert who first used the phrase 'Internet of Things' in a presentation in 1999 [1]. Neil Gross, speaking to Business Week commented, "In the next century, planet earth will don an electronic skin. It will use the Internet as a scaffold to support and transmit its sensations. This skin is already being stitched together. It consists of millions of embedded electronic measuring devices: thermostats, pressure gauges, pollution detectors, cameras, microphones, glucose sensors, EKG's, electroencephalographs. These will probe and monitor cities and endangered species, the atmosphere, our ships, highways and fleets of trucks, our conversations, our bodies-even our dreams." There are other phrases that are used instead of Internet of Things Phrase. Some are Internet of Objects, Embedded Intelligence, Web of Things, Pervasive Computing, Ubiquitous Computing, Internet of Connected Devices, Cyber Physical Systems, Human Computer Interaction, Wireless Sensor Networks etc. Although many phrases are used interchangeably the idea of IoT is exactly identical from general perspective. Definitions of IoT in literature are also diverse. Kevin Ashton defines IoT as a grid in which things in the physical world could be linked to the Internet via sensors. Mamdakam et al. describes IoT as an open and comprehensive network of intelligent objects which are capable of self-organizing and data sharing and those intelligent objects which have ability of reacting in any changes happening in our environment [2]. According to The Internet Architecture Board (IAB) IoT is a technology that a large number of embedded devices which are not directly on human control such as "smart objects" employ Internet Protocols Communication Services. Encyclopedic dictionary defines the IoT as the interdependence of everyday objects with each other and connection of these objects to internet to able to send and receive information [3]. Another definition which is made by IEEE is that IoT is a system in which everything is represented and presented in the Internet. According to IEEE IoT enables the interactions between things and application in cloud. Atzori et al. [4] describes IoT in three approaches: 1 . Internet-oriented which is represented by middleware 2 . Things oriented which is represented by sensors 3. Semantic-oriented which is represented by knowledge. Atzori also claims that these three paradigms should intersect to define IoT truly. Another definition that is made by Belissent [5] is that IoT is an environment which takes advantages of information and communication technologies to realize essential system elements and services for a public transformation, city utilities, city administration, public health and safety and more. Different and long definitions made for IoT does not mean that there is a disagreement about explanation of IoT, on the contrary all the definitions show different aspects of IoT. Hence to make complete definition and explanation for IoT all these definitions should be considered together. That is why there is no unique definition of IoT.

Looking at the Internet World Statistics there are more than 201 countries that use internet and 3,731,973,423 internet users by March 2017 [6]. This reveals that $\% 49$ of the world's 
population is using internet. Figure 1 shows internet users in the world by regions by March 2017.

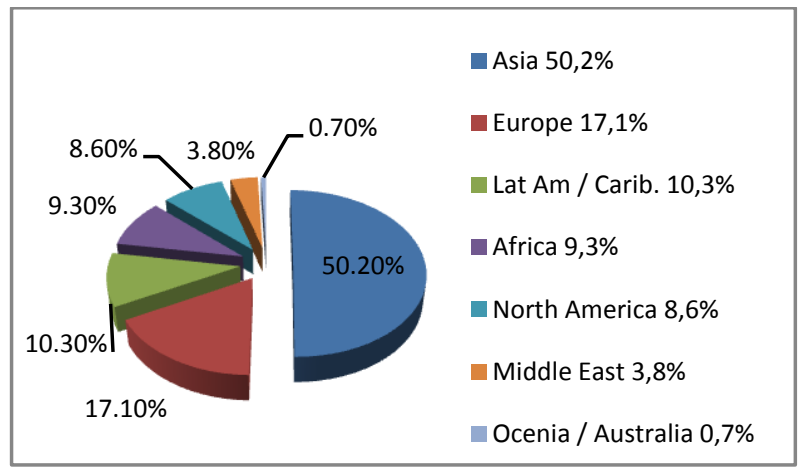

Fig 1: Internet Users in the World by regions, March 25, 2017, Source: Internet World Stats

Interpreting the statistics of internet users, considering the effects of internet and the ability of objects surrounding the world it is not hard to say that most of the objects around us will be on the internet in different forms. Internet, wireless sensor networks, Wi-Fi, 4G-LTE, IPv6, EPC, NFC, Sensors, telephonic data services and RFID technologies are precursory technologies to realize this idea. To give an overall example, the number of equipment which are connected to each other started to surpass the real number of people on earth only in 2011[7]. There exist more than 6.4 billion interconnected devices excluding smart phones, tablets and computers at the present and this matches up with Gartner's estimate [8]. According to International Data (IDC) Corporation the number of interconnected devices is 9 billion excluding tablets, smartphones and computers for now. According to Information Handling Services (IHS) this number is 17.6 billion including smart phones, tablets and computers. What about future? Chief Technology Officer of Stringify estimates 30 billion devices by 2020, Ericsson expects 28 billion by 2021, IHS markit claims 30.7 billion by 2020 , and Gartner's expectation is 20.8 billion by 2020 . All these numbers, estimates and claims can change from person to person however the reality which does not change from person to person is the evolution and revolution of IoT.

Figure 2 shows a graph of Google Trends that shows IoT interest over time worldwide from May 6, 2012 to May 6, 2017. Numbers represent search interest relative to the highest point on the chart for the given region and time. A value of 100 is the peak popularity for the term. A value of 50 means that the term is half as popular. Likewise a score of 0 means the term was less than $1 \%$ as popular as the peak. For May 6 , 2017 popularity for the IoT term is 68 [9].

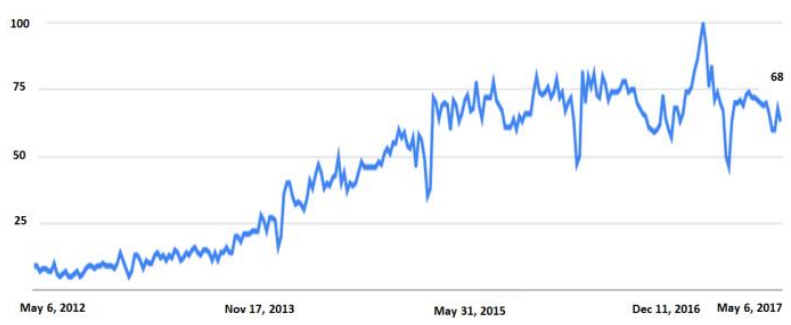

Fig 2 : Interest Over Time, https://trends.google.com/trends

The interconnected devices depend mainly on the sensors to sense changes around the environment and actuators to prompt, track, identify, control, automate and monitor some actions such as opening a door, activating a motor, closing a lamp, lifting an elevator, warning a user, lowering the speed of a car etc. A very simple example of IoT application which is already available in our houses or offices is a simple thermostat which changes heating or cooling level, lightning level depending on temperature or overcast weather. Figure 3 demonstrates IoT emerging trends and potential opportunities. Ubiquitous computing and pervasive computing have very powerful relationship with IoT idea. However one quite remarkable difference is that ubiquitous and pervasive computing relies on connecting one single device to a telecommunications administrative centre whereas IoT relies on connecting many devices to a telecommunications administrative centre and to each other, a communication between human-to-human, human-to-things, things-to-things [10]. One important thing about IoT is that the word 'Thing' does not necessarily stand for only electronic devices like servers, computers, tablets, telephones and smarts phones but also any 'Thing' which can be discernable by the real world. This adds up to things can be living things such as people, animals, vegetables, fruits, plants and non-living things like desk, seat, paper, pen, wall, window, slipper, curtain etc.

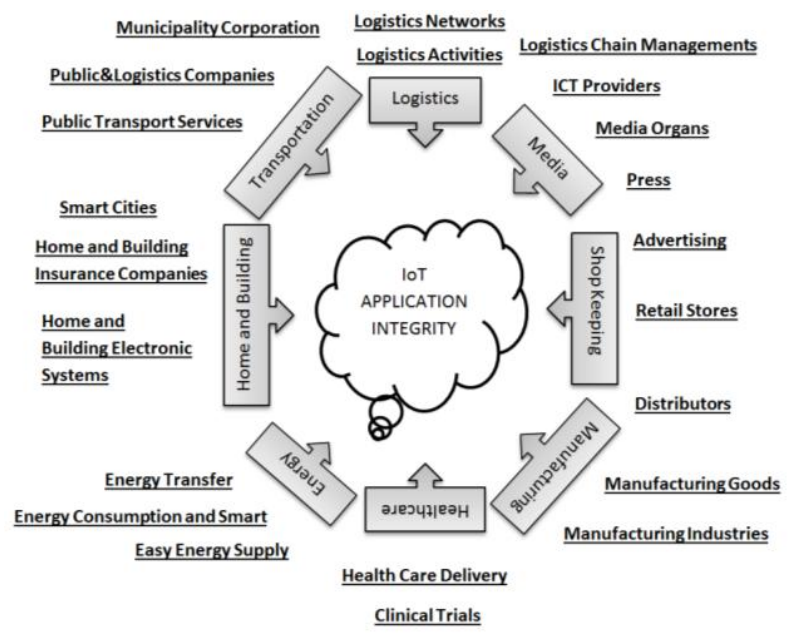

Fig 3: IoT Emerging Trends and Potential Opportunities

\section{HISTORY, LITERATURE SURVEY AND CHALLANGES}

Although IoT was born between 2008 and 2009 the IoT idea is not new. Besides, key IoT devices such as semiconductors, sensor and remote access services have been used for a quite long time. What is new is that the action, the idea is growing and now beginning to deploy. Table 1 is a key critical and turning points of IoT advancements.

The article entitled 'The Internet of Things' in Forbes Magazine in 2002 is considered to be the first documented use of the term IoT [16], [25], [28], [29] and the book entitled 'When Things Start to Think' by Neil Gernshenfeld is the first published book in 1999 [15], [16]. In recent past, on the other hand IoT term deployed rapidly. In 2008 for example, the first scientific and academic conference was held [30]. Feller [31] examined the ability of computation, communication, and control technologies to improve human interaction with pets using IoT phenomena. Mattern et al. [25] presented a survey called 'From the Internet to the Internet of Things' by considering up-to-date and significant technological developments like RFID, wireless sensor networks, web 
Table 1. From Its First Glimpses into Today's Trending Topic

\begin{tabular}{|c|c|c|c|c|}
\hline Technology & By & Country & Reference & Year \\
\hline Internet Connected Coke Vending Machine & Carnegie Melon University & USA & [11] & 1982 \\
\hline Ubiquitous term was first coined & Mark Weiser & USA & [12] & 1988 \\
\hline Deluxe Automatic Radiant Control Toaster & John Romkey and Simon Hacket & Australia & [13] & 1990 \\
\hline Tangible Telephone & Scott Brave and Hiroshii Ishii & USA & {$[14]$} & 1998 \\
\hline First IoT touch, Water Fountain & Mark Weiser & USA & [12] & 1998 \\
\hline IoT term is first coined & Kevin Ashton & UK & [1] & 1999 \\
\hline First book was written about IoT & Neil Gershenfeld & USA & {$[15]$} & 1999 \\
\hline EPC development & Auto-ID Labs & USA & [16] & 2003 \\
\hline First Internet of Refrigerator Plans & LG & $\begin{array}{l}\text { South } \\
\text { Korea } \\
\end{array}$ & [17] & 2000 \\
\hline The Ambient ORB & David Rose & USA & [18] & 2002 \\
\hline IoT term first used in Main Stream Publications & US Department of defense & USA & [19] & 2003 \\
\hline WiFi Enabled Artificial Rabbit, Nabaztag & Rafi Haladjian and Olivier Mével & France & [20] & 2005 \\
\hline ARDUINO Microcontroller invented & Interaction Design Institute Ivrea & Italy & [21] & 2005 \\
\hline IPSO Alliance, use of IP in networks of Smart Objects & IPSO Alliance & Global & [22] & 2008 \\
\hline Use of White Space Spectrum & $\begin{array}{l}\text { Federal Communications } \\
\text { Commisions }\end{array}$ & USA & [23] & 2008 \\
\hline IoT accepted as Disruptive Civil technologies & US National Intelligence Council & Global & {$[24]$} & 2008 \\
\hline More objects connected to internet than people & $\begin{array}{l}\text { CISCO Business Solutions } \\
\text { Group } \\
\end{array}$ & Global & {$[2]$} & 2009 \\
\hline IPv6 Public Launch & Internet Engineering Task Force & Global & [24] & 2011 \\
\hline INTEL launched Internet of Things Solution Groups & INTEL & USA & {$[25]$} & 2013 \\
\hline A Pilot Smart City Implementation, Padova Smart City & University of Padova & Italy & {$[26]$} & 2014 \\
\hline Self-driving Car & TESLA's Elon Musk & USA & [27] & 2017 \\
\hline
\end{tabular}

when it comes to reality. Yang et al. [32], [33] were another researchers who studied on security, privacy, limitations of IoT devices, IoT attacks, mechanisms and architectures for authentication and access control. Farooq et al. [34] is a good source for the researcher who are interested IoT enabling technologies and architectures of IoT. Swarnamugi et al. [35]studied on IoT technologies and Machine Learning Algorithms by investigating how machine-learning algorithms are applied to IoT data classification and prediction. Between 2008 and 2017 a lot of scientific projects rose to the surface by different important groups and companies. Some of these projects are: The European Technology Platform on Smart Systems Integration Project (ETP EPoSS, 2008), Berkeley University Cyber Physical Systems Project (2008), Coordination and Support Action for Global RFID-related Activities and Standardization Project, (CASAGRAS Project, 2009), The Cluster of European Research Projects on the Internet of Things, (CERP-IoT, 2010), Internet of Things Architecture Project (IoT-A, 2011), IoT European Research Cluster Project, (IERC, 2014), Internet Connected Objects for Reconfigurable Ecosystems Project, (iCore, 2013), PADOVA Smart City Project (2014) [16], [26].
Advancements about IoT spread out in 2015 and 2016. Here is a fascinating list of IoT news in 2015 and 2016. For the year 2015: in February, SIGFOX announced [36] to build the first IoT based telecommunication network known as Low Power Wide Area Network (LPWAN). In March, International Business Machine (IBM) excited the all world by announcement \$3 Billion Budget on a new IoT company. In March again, AMAZON got quite serious about IoT by getting 2lementry IoT platform. In April, the wearable company JAWBONE got $\$ 300$ Million which is the largest of all IoT 2015 start-up funding rounds. In May, GOOGLE announced IoT projects Weave and Brillo operating system. In June, wearable company FITBIT climbed after IBO. In August, chipmaker Qualcomm acquired CSR to boost its Bluetooth chip offering in segments such as wearables and smart home [37]. In September, US Government announced Smart Cities Initiatives with a $\$ 160$ Million budget. For the year 2016: in February, CISCO declared to buy IoT platform Jasper Technologies for $\$ 1.4$ Billion as a next step for CISCO's 'Internet of Everything'. In March, the number of IoT platforms exceeded more than 360. In May, European Union passed a new data protection policy called 'General Data Protection Regulation (GDPR)' which has a wide range 
of possibilities such as; reporting security breaches, providing explicit of data being processed, not processing the data which comes from IoT devices that children send and providing each user to object automated decision making. In July, SOFTBANK declared to takeover chipmaker ARM for a $\$ 32$ Billion budget. In September, the first IoT devices based large-scale cybersecurity attack (DDoS) shut down Dyn servers which allows the hacker to connect IoT infected devices. In October, US-based chipmaker Qualcomm announced to buy the leader in connected car chips NXP for $\$ 39 \mathrm{~B}$, making it the biggest-ever deal in the semiconductor industry [16]. When coming to the ends of October 2016, undoubtedly the consumer IoT success story of the year was reported by AMAZON Smart Home Voice Control. Amazon sold more than 1 million Echo devices in December 2016.

\subsection{Challenges}

IoT is a trend topic with its perils as much as its advantages. A lot of obstacles rose with internet when it started to develop and deploy. It is quite obvious that the same and more obstacles will occur with IoT as it starts to grow. Is IoT going to create the dark ages for humanity in terms of security and privacy violations, surveillance, consumer rights? How will humanity interact with IoT environment in their personal, social and economic lives? These significant challenges can be so hazardous that it can constitute an impediment on the way of realizing its potential benefits. For the present moment the challenges and doubts about IoT technology can be summarized as follows [16], [33];

$>$ Security and Privacy Issues: Although security and privacy considerations are general challenges of Information Technologies the new applications and attributes of IoT creates new diverse security and privacy problems. The first priority should be about identifying these problems. Information Technology companies and developers should be aware of the fact that each IoT user in the world wants to trust technology in security and privacy point of view. Any technology with an inefficient security is on the target of cyber-attacks and thefts. The most charming of Information Technologies becomes the user comfortability expectations and confidence after many privacy violated events.

$>$ Interoperability and Standards: Interoperability is defined as the capability of a product, a service, a system or different forces to interact, to communicate, to exchange information and to function with each other. IoT interoperability, configuration, designation and standardization are still not elaborated yet. Therefore IoT interoperability and standards is a big field for researchers who are involved in IoT challenges.

$>\quad$ Legal Rights and Regulatory Affairs: Legal, Rights and Regulatory of IoT devices are maybe the most complicated challenges from governments' point of view. As the each country adopts IoT technology each country will constitute its own rules regulations. This will create a worldwide concern because of a wide infrastructure of IoT. A common sense and a general shared wisdom for all governments are needed to be created. For this of course all the countries must find the least common denominator.

$>$ Related Economy and Advancement Issues: According to McKinsey Global Institute IoT has a total potential economic impact of $\$ 3.9$ trillion to
$\$ 11.1$ trillion per year in 2025 [38]. How will equal distribution be achieved for all part of the world because it is believed that IoT should be a tool for global empowerment without looking at consumer's location, region, country or economic development level?

$>$ Scalability: IoT capability of application, standards and services to develop and extend is unpredictable for the present time. More than estimated researches should be conducted relating to the performance and cost in response to changes in throughput or demand.

$>$ Operational Control Issues and Fault Tolerance: The world of things is much more vigorous and changeable than the world of computers. Moreover operation and control of IoT based smart objects should not be thought as simple as those of internet of computers because of complexity of smart objects.

$>$ Software Complexity: a possible infrastructure needed for a smart environment makes the software of IoT quite complex to an immeasurable extend.

$>$ Data Volumes and Data interpretation: It is easy to predict problems related to these issues considering the all IoT devices such as sensors, actuators, networks, data being produced etc. Common developments and mutual studying of IoT and Big Data is going to be heart of data volumes and interpretation challenges.

$>$ Power Needs: An IoT phenomenon offers a limitless involvement of devices which correspondingly needs a limitless power supply. IoT devices will be used in environments where charging does not exist. Their energy to execute the designate functionality is limited. Some solutions exist to overcome this energy problem. The first solution is to increase battery capacity but most of the IoT devices are designed in small size and they should be lightweight therefore there is no extra room for larger battery [32]. The critical question is: will the devices be able to produce the power they needed?

\section{METHODS AND MATERIALS: IOT ARCHITECTURE}

IoT does not have a single universally consensus on the IoT architecture. For the last decades various proposals for IoT architecture have been presented. Main IoT architecture proposes three- and- five layered architecture [10], [39]-[42]. Three- layered architecture consists of perception layer, network layer and application layer. Five- layered architecture adds processing layer and business layer to three- layered architecture. Ning and Wang [43] made use of human brain processing layers to develop IoT architecture. They were inspired by modified manlike neural network (MLN), which is the ability of human to see, smell, think, examine, remember, feel, make a decision and respond to physical environment. According to Ning and Wang human brain processing phenomena is very similar to IoT technology now that they are both a kind of complicated intelligent system which can see, taste, feel and control things, or even make decisions. Human brain can be seen as processing unit or data center. Spinal cord can be thought as data processing nodes distributed network which is shortly smart gateways [44]. Network of nerves can be thought as networking components, actuators and sensors of IoT. 


\subsection{Coding Layer}

Coding layer is the first IoT layer which gives identification to each of IoT object. In this layer, each object is assigned a unique ID which makes it easy to discern the objects [34], [45].

\subsection{Perception layer (Device Layer)}

Perception layer which is called sensing layer as well is the lowermost layer whose main characteristic is to sense environmental changes using sensors. Perception layer is known as sense organs of IoT that perceive, sense and detect so many environmental changes such as temperature, pressure, humidity, radiation, light, sound, smell, mobility, location, speed, acceleration of the objects around, collect overall useful data and convert these analog signals to digital signals. Then this digital information is passed to the next layer, Network Layer, for further processing. IoT devices connect to internet or each other via Ethernet connection, WiFi connection, GSM, WiMaX, 3G, ZigBee gateway, Bluetooth so on.

\subsection{Network Layer (Communication}

\section{Layer)}

IoT devices are connected to each other or to clouds via network layer. Network layer needs some communication protocols like, HTTP/HTTPS, MQTT 3.1/3.1.1, Constrained Application Protocol (CoAP), IPv4, IPv6, DDS and so on to connect devices. Network layer transmits information from perception layer to middleware layer (information processing system) safely.

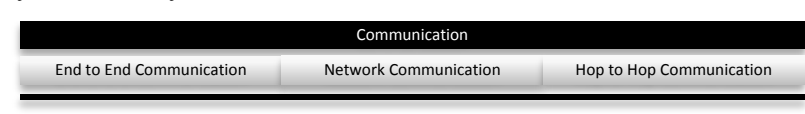

Fig 4: Network Layer

\subsection{Middleware Layer (The Event Processing and Analytics Layer)}

Middleware layer is a software interface layer between the physical layer and the application layer. This important layer disconnects users and developers from the exact knowledge of

the heterogeneous set of technologies adopted by the lower layers. It provides the required abstraction to hide the heterogeneity and the complexity of the underlying technologies involved in the lower layers [46]. Each device in IoT applications connect and communicate with just some devices which are involved in the same application. The layer which is responsible for service management of these devices is middleware layer which has link to database. Middleware layer receives data from network layer and keep this data in database. It processes the information and performs ubiquitous computation. Based on the results of processing, the layer takes automatic.

\subsection{Application Layer}

Application layer deals with application as the name implies. This top layer delivers different applications to IoT users. These applications can be such diverse as logistics, transportation, home and building, energy, healthcare, manufacturing, retail, media etc.

\subsection{Business Layer}

Business layer manages the whole process from an industrial point of view by observing overall behavior of IoT applications and services. The information received from application layer is gone under business intelligence for benefits identifications. Performance evaluation is done over the output of each layer in order to enhance the services. Business Layer is where the IOT applications are integrated into business processes and enterprise systems.

Table 2. General Requirements for IoT Reference Architecture

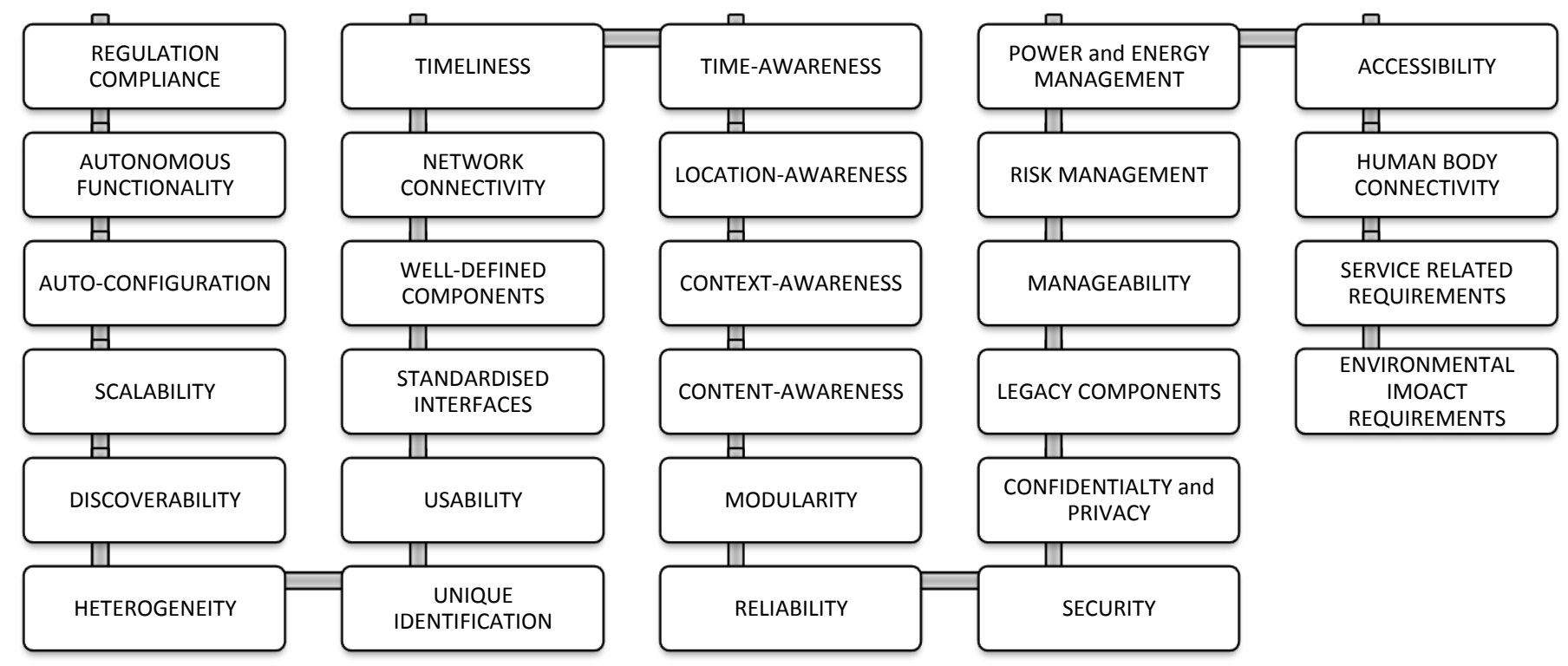


Table 3. Popular Architectures

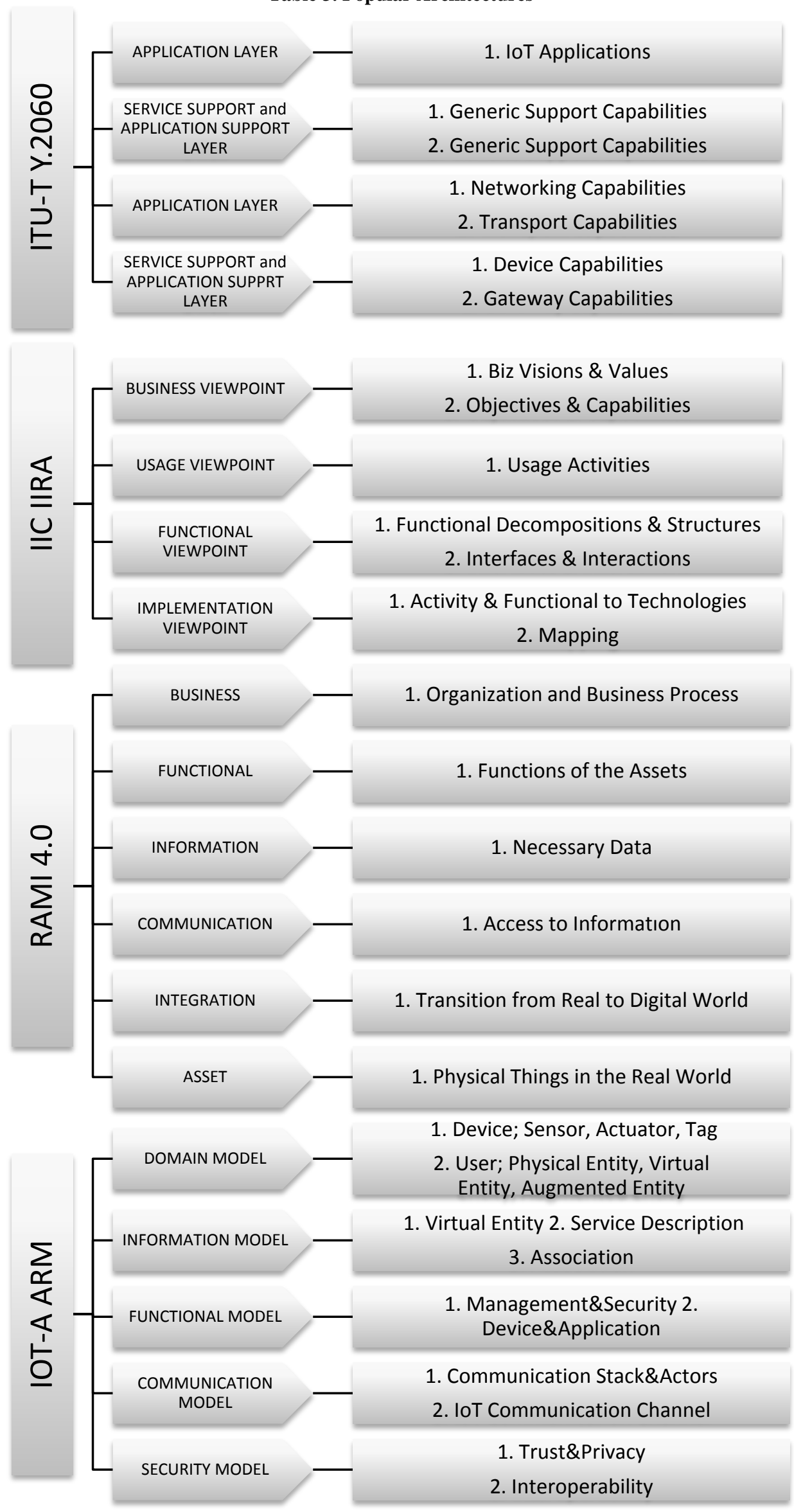


In addition to reference architecture, several standards organizations have constituted a number of IoT architectures/reference architectures. Table 3 shows these popular architectures in detail. The architecture models are divided into a number of layers. These architecture models will help managing the efforts of future IoT architects. The architectures specify IoT reference models which may consist of domain, information, functional, communication and security models, functional characteristics such as integration, safety, interconnectivity, object-related services, huge scale and heterogeneity and lastly high level requirements such as interoperability, full autonomous networking, identity-driven connectivity, security and privacy protection etc.

\section{CONCLUSION}

In the very near future most of the objects around us will be on the internet in various forms. In this paper the most up-todate emerging trends and potential opportunities of IoT are presented. A fascinating list of IoT news is demonstrated for the very recent past. The latest world statistics about IoT technologies are reported. Quite popular and charming IoT scientific projects are described. Developments and ongoing projects conclude that IoT is going to lead forth interconnection of objects with people at an unpredictable scale and level just as or probably more than internet did. Throughout of the paper we try to give critical and turning points of IoT. A general IoT architecture is proposed and well-liked architectures are described. IoT is mysterious with its promises as much as its doubts. Therefore significant possible IoT challenges are discussed and possible suggestions are proposed. Possible answers to very popular IoT related questions are discussed such as: Is IoT going to create the dark ages for humanity in terms of security and privacy violations, surveillance, and consumer rights? How will humanity interact with IoT environment in their personal, social and economic lives? Although it is never known what the future brings to our lives, this paper tries to make our future scenarios quite clear and visible by presenting the rapid IoT developments and by showing the promising ongoing IoT projects. To the best of our knowledge contributions of this paper are unique. Therefore we believe that this research paper is a comprehensive reference source for those who are interested in IoT and smart objects.

\section{REFERENCES}

[1] K. Ashton, "That 'Internet of Things' Thing," RFiD J., p. 4986, 2009.

[2] S. Madakam, R. Ramaswamy, and S. Tripathi, "Internet of Things (IoT): A Literature Review," J. Comput. Commun., vol. 3, no. 3, pp. 164-173, 2015.

[3] “Internet of Things," Oxford Dictionaries. 2015.

[4] L. Atzori, A. Iera, and G. Morabito, "The Internet of Thinags: A survey," Comput. Networks, vol. 54, no. 15, pp. 2787-2805, 2010.

[5] J. Bélissent, "Getting Clever About Smart Cities : New Opportunities Require New Business Models," Forrester Research, Cambridge, 2010.

[6] "Internet Users in the World by regions," 2017. [Online]. Available: http://www.internetworldstats.com/stats.htm.

[7] J. Gubbi, R. Buyya, and S. Marusic, "Internet of Things ( IoT ): A Vision, Architectural Elements, and Future Directions," Futur. Gener. Comput. Syst., vol. 29, no. 1, pp. 1-19, 2013.
[8] “Gartner's Estimate," 2017. [Online]. Available: http://www.gartner.com/newsroom/id/3598917.

[9] "IoT Google Trends." [Online]. Available: https://trends.google.com/trends/.

[10] V. R. Vedang and S. Gopal, "A Literature Review on Internet of Things (IoT)," Int. J. Comput. Syst., vol. 2, no. 8, pp. 355-358, 2015.

[11] Carnegie-University-Mellon-Computer -Science, "The 'Only' Coke Machine on the Internet," Web. Carnegie Mellon University Computer Science Department, n.d. Web. 06 Sept. 2015.

[12] "In Memory of Dr. Mark Weiser," PARC. [Online]. Available: $\quad$ http://www.parc.com/services/focusarea/ubicomp/.

[13] "First Internet of Things Toaster." [Online]. Available: https://en.wikipedia.org/wiki/Simon_Hackett.

[14] S. B. Brave and S. A. Benton, "Tangible Interfaces for Remote Communication and Collaboration," Massachusetts Institute of Technology, 1998.

[15] N. Gershenfeld, When Things Start to Think. Henry Holt and Co., Inc., New York, NY., 1999.

[16] D. R. R. Minerva, A. Biru, "IEEE IoT Towards Definition Internet of Things Rev. 1," IEEE Internet Things, pp. 1-86, 2015.

[17] U. N. S. E. F. (UNSEF), "A Brief History of the IoT," in Workshop on Internet of Things Development for the Promotion of Information Economy, 2015, pp. 1-12.

[18] J. Compton, "The Ambient Orb: Striving for a PC-Free Tomorrow," Yahoo! Voices, 2014.

[19] "Internet of Things History." [Online]. Available: https://www.postscapes.com/internet-of-things-history/.

[20] "Nabaztag." [Online]. Available: nabaztag.com.

[21] C. Rajan, B. Megala, A. Nandhini, and C. R. Priya, "A Review: Comparative Analysis of Arduino Micro Controllers in Robotic Car," vol. 9, no. 2, pp. 371-380, 2015.

[22] A. K. P. J. Vasseur, C. P. Bertrand, F. Watteco, B. Aboussouan, V. Marketing, G. E. Gnoske, "A survey of several low power Link layers for IP Smart Objects," Internet Protocol for Smart Objects (IPSO) Alliance, 2010.

[23] O. Kharif, "FCC's Kevin Martin Supports Freeing White Spaces," Business Week, 2008.

[24] K. Claffy, “Tracking IPv6 Evolution : Data We Have and Data We Need," ACM Comput. Commun. Rev., vol. 41, no. 3, pp. 43-48, 2011

[25] M. Friedemann and C. Floerkemeir, "From the Internet to the Internet of Things," From Act. Data Manag. to Event-Based Syst. More, pp. 242-259, 2011.

[26] A. Zanella, N. Bui, A. Castellani, L. Vangelista, and M. Zorzi, "Internet of Things for Smart Cities," IEEE Internet Things J., vol. 1, no. 1, pp. 22-32, 2014.

[27] S. Adario, "Elon Musk thinks universal income is answer to automation taking human jobs," mashable.com, 2017. 
[28] C. R. Schoenberger, "The internet of things," Forbes Magazine, 2002.

[29] D. R. R. Minerva, A. Biru, "IEEE IoT Towards Definition Internet of Things Rev. 1," IEEE Internet Things, vol. Revision1, pp. 1-86, 2016.

[30] S. E. S. C. Floerkemeier, M. Langheinrich, E. Fleisch, F. Mattern, "The Internet of Things," in First International Conference, IOT 2008, LNCS 4952, Springer, 2008.

[31] G. Feller, "The Internet of Things: In a Connected World of Smart Objects," Accent. Bankinter Found. Innov., 2011.

[32] Y. Yang, L. Wu, G. Yin, L. Li, and H. Zhao, "A Survey on Security and Privacy Issues in Internet-of-Things," IEEE Internet Things J., vol. PP, no. 99, p. 1, 2017.

[33] K. Rose, S. Eldridge, and C. Lyman, "The internet of things: an overview," Internet Soc., no. October, p. 53, 2015 .

[34] M. U. Farooq and M. Waseem, "A Review on Internet of Things ( IoT ),” Int. J. Comput. Appl. (0975 8887), vol. 113, no. 1, pp. 1-7, 2015.

[35] V. I. M. Swarnamugi, R.Chinnaiyan, "IoT Technologies and Machine Learning Algorithms - A Study," Int. J. Eng. Sci. Res. Technol., vol. 5, no. 10, pp. 614-621, 2016.

[36] R. D. G. Dregvaite, "Information and Software Technologies," in Information and Software Technologies: 22nd International Conference, ICIST Druskininkai, Lithuania, Proceedings. Springer.-. ISBN 978-3-319-46254-7., 2016, p. 665.

[37] C. Qualcomm, "Wearable and Smart Homes," 2015.
[38] J. Manyika et al., "The Internet of Things: Mapping the value beyond the hype," McKinsey Glob. Inst., no. June, p. 144, 2015.

[39] I. Mashal, O. Alsaryrah, T. Y. Chung, C. Z. Yang, W. H. Kuo, and D. P. Agrawal, "Choices for interaction with things on Internet and underlying issues," Ad Hoc Networks, vol. 28, pp. 68-90, 2015.

[40] O. Said and M. Masud, "Towards internet of things: Survey and future vision," Int. J. Comput. Networks, vol. 5, no. 1, pp. 1-17, 2013.

[41] M. Wu, T. J. Lu, F. Y. Ling, J. Sun, and H. Y. Du, "Research on the architecture of Internet of Things," ICACTE 2010 - 2010 3rd Int. Conf. Adv. Comput. Theory Eng. Proc., vol. 5, pp. 484-487, 2010.

[42] R. Khan, S. U. Khan, R. Zaheer, and S. Khan, "Future internet: The internet of things architecture, possible applications and key challenges," Proc. - 10th Int. Conf. Front. Inf. Technol. FIT 2012, pp. 257-260, 2012.

[43] H. Ning and Z. Wang, "Future Internet of Things Architecture :," IEEE Commun. Lett., vol. 15, no. 4, pp. 461-463, 2011.

[44] P. Sethi and S. R. Sarangi, "Internet of Things: Architectures, Protocols, and Applications," J. Electr. Comput. Eng., vol. 2017, pp. 1-25, 2017.

[45] S. K. Chaulya and G. M. Prasad, Sensing and Monitoring Technologies for Mines and Hazardous Areas: Monitoring and Prediction Technologies, 1. Edition. Cambridge: Elsevier, 2016.

[46] D. T. I. R. M. R. Abdmeziem, “Architecting the Internet of Things: State of the Architecting the Internet of Things : State of the," Robot. Sens. Clouds. Stud. Syst. Decis. Control. Springer, Cham, vol. 36, no. July, pp. 55-75, 2015.

[47] 